\title{
Different Approximations to the Solution of Upper-Convected Maxwell Fluid over a Porous Stretching Plate
}

\author{
Vasile Marinca, ${ }^{1,2}$ Remus-Daniel Ene, ${ }^{3}$ Bogdan Marinca, ${ }^{4}$ and Romeo Negrea ${ }^{3}$ \\ ${ }^{1}$ Department of Mechanics and Vibration, University Politehnica Timişoara, 300222 Timişoara, Romania \\ ${ }^{2}$ Department of Electromechanics and Vibration, Center for Advanced and Fundamental Technical Research, \\ Romania Academy, 300222 Timişoara, Romania \\ ${ }^{3}$ Department of Mathematics, University Politehnica Timişoara, 300006 Timişoara, Romania \\ ${ }^{4}$ Department of Applied Electronics, University Politehnica Timişoara, 300223 Timişoara, Romania
}

Correspondence should be addressed to Remus-Daniel Ene; eneremus@gmail.com

Received 14 March 2014; Revised 29 May 2014; Accepted 29 May 2014; Published 6 July 2014

Academic Editor: Dragos-Patru Covei

Copyright (C) 2014 Vasile Marinca et al. This is an open access article distributed under the Creative Commons Attribution License, which permits unrestricted use, distribution, and reproduction in any medium, provided the original work is properly cited.

In the present paper, we consider an incompressible magnetohydrodynamic flow of two-dimensional upper-convected Maxwell fluid over a porous stretching plate with suction and injection. The nonlinear partial differential equations are reduced to an ordinary differential equation by the similarity transformations and taking into account the boundary layer approximations. This equation is solved approximately by means of the optimal homotopy asymptotic method (OHAM). This approach is highly efficient and it controls the convergence of the approximate solutions. Different approximations to the solution are given, showing the exceptionally good agreement between the analytical and numerical solutions of the nonlinear problem. OHAM is very efficient in practice, ensuring a very rapid convergence of the solutions after only one iteration even though it does not need small or large parameters in the governing equation.

\section{Introduction}

The flow of non-Newtonian fluids has been analyzed by numerous researchers, because, in practical applications, non-Newtonian fluids are more appropriate than Newtonian fluids. For such fluids, the Navier-Stokes theory becomes inadequate and obviously there are several constitutive equations that have been proposed to various types of nonNewtonian fluids. Examples of the flow of non-Newtonian fluids occur in a large variety of applications: synthetic fibres, drilling muds, food processing, plastic polymers, and so on. There are numerous constitutive equations that have been proposed to various types of non-Newtonian fluids; a majority of non-Newtonian fluids models are concerned with the fluids of grade two or three. One type of fluids in which the relaxation type phenomena can be considered is known as Maxwell model. Some investigations in this field are made by the mathematicians, engineers, physicians, and computer scientists. Sakiadis $[1,2]$ first studied various aspects of the stretching problem: the flow due to a semi-infinite horizontally moving wall in an ambient fluid. Phan-Thien [3] considered the plane and axisymmetric stagnation flows in a Maxwell fluid, using the shooting and boundary element method, like Zheng et al. [4]. Sadeghy et al. [5] considered the problem of hydrodynamic Sakiadis flow of an upperconvected Maxwell fluid over a rigid plate moving steadily in an otherwise quiescent fluid. Homotopy analysis method is used by Hayat et al. [6] to solve nonlinear differential equation of the upper-convected Maxwell fluid over a porous stretching plate. Also, the same method is considered by Hayat and Sajid [7], Abbas et al. [8, 9], and Hayat et al. [10]. Dual solutions in mixed convection flow near stagnation point on a vertical porous plate have been presented by Ishak et al. [11]. Sahoo [12] investigated the effects of partial slip in the MHD flow and mass transfer of an electrically conducted second grade fluid past an axisymmetric stretching sheet.

Taking into considerations these analyses, the objective of the present paper is to propose an accurate procedure to nonlinear differential equation of the magnetohydrodynamic flow of an upper-convected Maxwell fluid over a porous 
stretching plate using OHAM. A version of the OHAM is applied in this study to derive highly accurate analytical expressions of the solutions. Our procedure does not depend upon any small or large parameters, contradistinguishing from other known methods in literature. The main advantage of this approach is the control of the convergence of approximate solutions in a very rigorous way. A very good agreement was found between our approximate solutions and numerical solutions, which proves that our method is very efficient and accurate.

\section{Equation of Motion}

If we consider the steady incompressible two-dimensional flow of an upper-convected Maxwell fluid over a porous stretching plate, then the constitutive equation for the Cauchy stress tensor $\mathbf{T}$ is given by

$$
\mathbf{T}=-p \mathbf{I}+\mathbf{S}
$$

such that the extra tensor $\mathbf{S}$ satisfies

$$
\mathbf{S}+\lambda\left(\frac{d \mathbf{S}}{d t}-\mathbf{L S}-\mathbf{S L}^{T}\right)=\mu \mathbf{A}_{1}
$$

where $\mu$ is the viscosity, $\lambda$ is the relaxation time, and the first Rivlin-Ericksen tensor $\mathbf{A}_{1}$ is defined as follows:

$$
\mathbf{A}_{1}=(\operatorname{grad} \mathbf{V})+(\operatorname{grad} \mathbf{V})^{T}
$$

in which $d / d t$ is the material time derivative and $\mathbf{V}$ is the velocity.

For the magnetohydrodynamic Sakiadis flow, by imposing a uniform magnetic field $B_{0}$ along the $y$-direction and neglecting the induced magnetic field, the equations which govern the steady flow can be written as

$$
\begin{gathered}
\frac{\partial u}{\partial x}+\frac{\partial v}{\partial y}=0 \\
\rho\left(u \frac{\partial u}{\partial x}+v \frac{\partial u}{\partial y}\right)=-\frac{\partial p}{\partial x}+\frac{\partial S_{x x}}{\partial x}+\frac{\partial S_{x y}}{\partial y}-\delta B_{0}^{2} u \\
\rho\left(u \frac{\partial v}{\partial x}+v \frac{\partial v}{\partial y}\right)=-\frac{\partial p}{\partial y}+\frac{\partial S_{y x}}{\partial x}+\frac{\partial S_{y y}}{\partial y}
\end{gathered}
$$

where $u, v$ are the velocity components, $p$ is the pressure, $\rho$ is the density, $\delta$ is the electrical conductivity, and $S_{x x}, S_{x y}, S_{y x}$, $S_{y y}$ are the components of the extra tensor $\mathbf{S}$.

Using the boundary layer approximations $[5,13]$

$$
\begin{gathered}
u=\mathcal{O}(1), \quad v=\mathcal{O}(\delta), \quad x=\mathcal{O}(1), \quad y=\mathcal{O}(\delta), \\
\frac{T_{x x}}{\rho}=\mathcal{O}(1), \quad \frac{T_{x y}}{\rho}=\mathcal{O}(\delta), \quad \frac{T_{y y}}{\rho}=\mathcal{O}\left(\delta^{2}\right),
\end{gathered}
$$

the flow in the absence of the pressure gradient is governed by (4) and

$$
\begin{aligned}
u \frac{\partial u}{\partial x} & +v \frac{\partial v}{\partial x}+\lambda\left(u^{2} \frac{\partial^{2} u}{\partial x^{2}}+v^{2} \frac{\partial^{2} u}{\partial y^{2}}+2 u v \frac{\partial^{2} u}{\partial x \partial y}\right) \\
& =v \frac{\partial^{2} u}{\partial y^{2}}-\frac{\delta B_{0}^{2}}{\rho} u
\end{aligned}
$$

where $v$ is the kinematic viscosity of the fluid.

The relevant initial/boundary conditions for the flow problem are

$$
\begin{aligned}
& u=C x, \quad v=-V_{0}, \quad \text { at } y=0 \\
& u \longrightarrow 0, \quad \text { as } y \longrightarrow \infty,
\end{aligned}
$$

in which $C$ is the stretching rate and $V_{0}>0$ is the suction velocity and $V_{0}<0$ is the injection velocity.

Introducing the stream function $\Psi$ such that

$$
u=\frac{\partial \Psi}{\partial y}, \quad v=-\frac{\partial \Psi}{\partial x},
$$

then (4) is identically satisfied and (7) becomes

$$
\begin{gathered}
\frac{\partial \Psi}{\partial y} \frac{\partial^{2} \Psi}{\partial x \partial y}-\frac{\partial \Psi}{\partial x} \frac{\partial^{2} \Psi}{\partial y^{2}}+\lambda\left[\left(\frac{\partial \Psi}{\partial y}\right)^{2} \frac{\partial^{3} \Psi}{\partial x^{2} \partial y}+\left(\frac{\partial \Psi}{\partial x}\right)^{2} \frac{\partial^{3} \Psi}{\partial y^{3}}\right. \\
\left.\quad-2 \frac{\partial \Psi}{\partial y} \frac{\partial \Psi}{\partial x} \frac{\partial^{3} \Psi}{\partial x \partial y^{2}}\right] \\
=v \frac{\partial^{3} \Psi}{\partial y^{3}}-\frac{\delta B_{0}^{2}}{\rho} \frac{\partial \Psi}{\partial y}
\end{gathered}
$$

Introducing the similarity transformations

$$
\eta=\sqrt{\frac{C}{v}} y, \quad \Psi=\sqrt{\nu C} x f(\eta),
$$

equation (9) becomes

$$
u=C x f^{\prime}(\eta), \quad v=-\sqrt{x} f(\eta) .
$$

Substituting (12) into (10), it obtains the governing equation in the following form:

$$
f^{\prime \prime \prime}-M^{2} f^{\prime}-f^{\prime 2}+f f^{\prime \prime}+\beta\left(2 f f^{\prime} f^{\prime \prime}-f^{2} f^{\prime \prime \prime}\right)=0,
$$

where $M^{2}=\delta B_{0}^{2} / \rho C$ and $\beta=\lambda C$.

Now, the initial/boundary conditions (8) are

$$
f(0)=R, \quad f^{\prime}(0)=1, \quad f^{\prime}(\infty)=0,
$$

with $R=V_{0} / \sqrt{\nu C}$, where $R>0$ corresponds to suction velocity and $R<0$ for injection velocity.

In what follows, the nonlinear differential equation (13) with initial/boundary conditions (14) can be solved analytically using OHAM. 


\section{Basic Ideas of the Optimal Homotopy Asymptotic Method}

Equation (13) with initial/boundary conditions (14) can be written in a more general form as follows:

$$
N[f(\eta)]=0,
$$

where $N$ is a given nonlinear differential operator depending on the unknown function $f(\eta)$, subjected to the initial/boundary condition

$$
B\left(f(\eta), \frac{d f(\eta)}{d \eta}\right)=0 .
$$

Let $f_{0}(\eta)$ be an initial approximation of $f(\eta)$ and $L$ an arbitrary linear operator such as

$$
L\left[f_{0}(\eta)\right]=0, \quad B\left(f_{0}(\eta), \frac{d f_{0}(\eta)}{d \eta}\right)=0 .
$$

It should be emphasized that this linear operator $L$ is not unique.

If $p \in[0,1]$ denotes an embedding parameter and $F$ is an analytic function, then we construct a homotopy [14-18]:

$$
\mathscr{H}\left[L(F(\eta, p)), H\left(\eta, C_{i}\right), N(F(\eta, p))\right], \quad i=1,2, \ldots, s
$$

with the properties

$$
\begin{aligned}
& \mathscr{H}\left[L(F(\eta, 0)), H\left(\eta, C_{i}\right), N(F(\eta, 0))\right] \\
& \quad=L(F(\eta, 0))=L\left(f_{0}(\eta)\right)=0, \\
& \mathscr{H}\left[L(F(\eta, 1)), H\left(\eta, C_{i}\right), N(F(\eta, 1))\right] \\
& \quad=H\left(\eta, C_{i}\right) N(F(\eta))=0, \quad i=1,2, \ldots, s,
\end{aligned}
$$

where $H\left(\eta, C_{i}\right) \neq 0$ is an arbitrary auxiliary convergencecontrol function depending on variable $\eta$ and on a number of arbitrary parameters $C_{1}, C_{2}, \ldots, C_{s}$ unknown now and will be determined later.

Let us consider the function $F$ in the form

$$
F(\eta, p)=f_{0}(\eta)+p f_{1}\left(\eta, C_{i}\right)
$$

By substituting (21) into equation obtained by means of homotopy (18),

$$
\mathscr{H}\left[L(F(\eta, p)), H\left(\eta, C_{i}\right), N(F(\eta, p))\right]=0, \quad i=1, \ldots, s
$$

and, then, equating the coefficients of $p^{0}$ and $p^{1}$, we obtain

$$
\begin{array}{r}
\mathscr{H}\left[L(F(\eta, p)), H\left(\eta, C_{i}\right), N(F(\eta, p))\right] \\
=L\left(f_{0}(\eta)\right)+p\left[L\left(f_{1}\left(\eta, C_{i}\right)\right)-L\left(f_{0}(\eta)\right)\right. \\
\left.-H\left(\eta, C_{i}\right) N\left(f_{0}(\eta)\right)\right]=0, \\
i=1,2, \ldots, s .
\end{array}
$$

From (23), we obtain the governing equation of $f_{0}(\eta)$ given by (17) and the governing equation of $f_{1}(\eta)$; that is,

$$
\begin{gathered}
L\left(f_{1}\left(\eta, C_{i}\right)\right)=H\left(\eta, C_{i}\right) N\left(f_{0}(\eta)\right), \\
B\left(f_{1}\left(\eta, C_{i}\right), \frac{d f_{1}\left(\eta, C_{i}\right)}{d \eta}\right)=0, \\
i=1, \ldots, s,
\end{gathered}
$$

where we find the following expression for the nonlinear operator:

$$
N\left(f_{0}(\eta)\right)=\sum_{i=1}^{m} h_{i}(\eta) g_{i}(\eta)
$$

where the functions $h_{i}(\eta)$ and $g_{i}(\eta), i=1, \ldots, m$, are known and depend on the function $f_{0}(\eta)$ and also on the nonlinear operator, $m$ being a known integer number.

In this way, taking into account (20), from (21), for $p=1$, we obtain the first-order approximate solution which becomes

$$
\bar{f}\left(\eta, C_{i}\right)=f_{0}(\eta)+f_{1}\left(\eta, C_{i}\right), \quad i=1, \ldots, s .
$$

It should be emphasized that $f_{0}(\eta)$ and $f_{1}\left(\eta, C_{i}\right)$ are governed by the linear equations (17) and (24), respectively, with boundary conditions that come from the original problem. It is known that the general solution of nonhomogeneous linear equation (24) is equal to the sum of general solution of the corresponding homogeneous equation and of some particular solutions of the nonhomogeneous equation. However, the particular solutions are readily selected only in the exceptional cases.

In what follows we do not solve (24), but, from the theory of differential equations, taking into considerations the method of variation of parameters, Cauchy method, method of influence function, the operator method [19], and so on is more convenient to consider the unknown function $f_{1}(\eta)$, in the form

$$
\begin{gathered}
f_{1}\left(\eta, C_{j}\right)=\sum_{i=1}^{n} H_{i}\left(\eta, h_{j}(\eta), C_{j}\right) g_{i}(\eta), \quad j=1, \ldots, s \\
B\left(f_{1}\left(\eta, C_{i}\right), \frac{d f_{1}\left(\eta, C_{i}\right)}{d \eta}\right)=0,
\end{gathered}
$$

where within expression of $H_{i}\left(\eta, h_{j}(\eta), C_{j}\right)$ appear linear combinations of some functions $h_{j}$, some terms which are given by the corresponding homogeneous equation, and the unknown parameters $C_{j}, j=1, \ldots, s$. In the sum, $\sum_{i=1}^{n} H_{i} g_{i}$ appear an arbitrary number of $n$ terms.

For instance, if $h_{1}=\sin \alpha \eta$, then we can choose $H_{1}\left(\eta, h_{1}, C_{j}\right)=C_{1} \sin \alpha \eta+C_{2} \cos \alpha \eta+C_{3} \sin 2 \alpha \eta+\cdots$. Similarly, if $h_{1}=\eta^{3}$, then we can choose $H_{1}\left(\eta, h_{1}, C_{j}\right)=$ $C_{1} \eta^{3}+C_{2} \eta+C_{3} \eta^{2}+C_{4} \eta^{4}+\cdots$. In the case when $h_{1}=\ln \eta$, we can choose $H_{1}\left(\eta, h_{1}, C_{j}\right)=C_{1} \ln \eta+C_{2} \ln ^{2} \eta+C_{3} \eta \ln \eta+\cdots$ or $H_{1}\left(\eta, h_{1}, C_{j}\right)=C_{1} \ln \eta+C_{2} \eta \ln \eta+C_{3} \eta^{2} \ln \eta+C_{4} \eta \ln 2 \eta+\cdots$. We have large freedom to choose the value of $n$. We cannot 
demand $f_{1}\left(\eta, C_{i}\right)$ to be solutions of $(24)$ but $\bar{f}\left(\eta, C_{i}\right)$, given by (26) with $f_{1}\left(\eta, C_{i}\right)$ given by (27), are the solutions of (15). This is an underlying idea of our method. The convergence of the approximate solution $\bar{f}\left(\eta, C_{i}\right)$ given by (26) depends upon the auxiliary functions $H_{i}\left(\eta, h_{i}, C_{j}\right), j=1, \ldots, s$. There are many possibilities to choose these functions $H_{i}$. We try to choose $H_{i}$ so that within (27) the term $\sum_{i=1}^{n} H_{i}\left(\eta, h_{j}(\eta), C_{j}\right) g_{i}(\eta)$ is of the same shape with the term $\sum_{i=1}^{m} h_{i}(\eta) g_{i}(\eta)$ given by (25). The first-order approximate solution $\bar{f}\left(\eta, C_{i}\right)$ also depends on the parameters $C_{j}, j=1, \ldots, s$. The values of these parameters can be optimally identified via various methods, such as the least-square method, the Galerkin method, the collocation method, and the Ritz method. The first option should be minimizing the square residual error:

$$
J\left(C_{1}, C_{2}, \ldots, C_{s}\right)=\int_{(D)} R^{2}\left(\eta, C_{1}, C_{2}, \ldots, C_{s}\right) d \eta,
$$

where the residual $R$ is given by

$$
R\left(\eta, C_{1}, C_{2}, \ldots, C_{s}\right)=N\left(\bar{f}\left(\eta, C_{i}\right)\right)
$$

The unknown parameters $C_{1}, C_{2}, \ldots, C_{s}$ can be identified from the conditions:

$$
\frac{\partial J}{\partial C_{1}}=\frac{\partial J}{\partial C_{2}}=\cdots=\frac{\partial J}{\partial C_{s}}=0
$$

With these parameters known (called optimal convergence-control parameters), the first-order approximate solution given by (26) is well determined.

It should be emphasized that our procedure contains the auxiliary functions $H_{i}\left(\eta, f_{i}, C_{j}\right), i=1, \ldots, m, j=$ $1, \ldots, s$, which provides us with a simple way to adjust and control the convergence of the approximate solutions. It is very important to properly choose these functions $H_{i}\left(\eta, f_{i}, C_{j}\right)$ which appear in the construction of the firstorder approximation.

\section{Different Approximations to the Solution of Upper-Convected Maxwell Fluid with OHAM}

In what follows we apply our procedure to obtain approximate solutions of (13) and (14). For this purpose, we choose the linear operator of the following form:

$$
L[f(\eta)]=f^{\prime \prime \prime}(\eta)-K^{2} f^{\prime}(\eta)
$$

We mention that the linear operator is not unique. Also, we have freedom to choose

$$
\begin{gathered}
L[f(\eta)]=f^{\prime \prime \prime}(\eta)+K f^{\prime \prime}(\eta), \\
L[f(\eta)]=f^{\prime \prime \prime}(\eta)+\frac{3 K}{K \eta+1} f^{\prime \prime}(\eta), \\
L[f(\eta)]=f^{\prime \prime \prime}(\eta)-\frac{6 K^{2}}{(K \eta+1)^{2}} f^{\prime}(\eta),
\end{gathered}
$$

where $K$ is an unknown positive parameter and will be determined later. The initial approximation $f_{0}(\eta)$ can be obtained from (17) with initial/boundary conditions

$$
f_{0}(0)=R, \quad f_{0}^{\prime}(0)=1, \quad f_{0}^{\prime}(\infty)=0 .
$$

Equation (17) with the linear operators (31) and (32) has the following solution:

$$
f_{0}(\eta)=R+\frac{1}{K}\left(1-e^{-K \eta}\right)
$$

while (17) with the linear operators (33) has the following solution:

$$
f_{0}(\eta)=R+\frac{1}{K} \ln (K \eta+1) .
$$

In what follows we consider only the linear operator given by (31) and the initial approximation given by (35).

The nonlinear operator corresponding to nonlinear differential equation (13) is defined by

$$
\begin{aligned}
N[f(\eta)]= & f^{\prime \prime \prime}-M^{2} f^{\prime}-f^{\prime 2}+f f^{\prime \prime} \\
& +\beta\left(2 f f^{\prime} f^{\prime \prime}-f^{2} f^{\prime \prime \prime}\right) .
\end{aligned}
$$

By substituting (35) into (37), it holds that

$$
\begin{aligned}
N\left[f_{0}(\eta)\right]= & {\left[K^{2}-M^{2}-1-K R-\beta(1+K R)^{2}\right] e^{-K \eta} } \\
& +\beta e^{-3 K \eta} .
\end{aligned}
$$

Comparing (25) and (38), one can get

$$
\begin{aligned}
& h_{1}(\eta)=K^{2}-M^{2}-1-K R-\beta(1+K R)^{2}, \\
& g_{1}(\eta)=e^{-K \eta}, \quad h_{2}(\eta)=\beta, \quad g_{2}(\eta)=e^{-3 K \eta} .
\end{aligned}
$$

The function $f_{1}(\eta)$ given by (27) becomes

$$
f_{1}\left(\eta, C_{i}\right)=H_{1}\left(\eta, C_{i}\right) e^{-K \eta}+H_{2}\left(\eta, C_{i}\right) e^{-3 K \eta},
$$

where we have the freedom to choose a lot of possibilities for the unknown functions $H_{i}$ as follows:

$$
\begin{aligned}
H_{1}\left(\eta, C_{i}\right)= & -\frac{C_{1}+C_{3}+C_{5}}{K}+C_{1} \eta+C_{2} \eta^{2} \\
& +\left(\frac{C_{1}+C_{3}+C_{5}}{K}+C_{3} \eta+C_{4} \eta^{2}\right) e^{-K \eta}, \\
& H_{2}\left(\eta, C_{i}\right)=C_{5} \eta+C_{6} \eta^{2} .
\end{aligned}
$$

Substituting (41) into (40), we have

$$
\begin{aligned}
f_{1}\left(\eta, C_{i}\right)= & -\left(\frac{C_{1}+C_{3}+C_{5}}{K}+C_{1} \eta+C_{2} \eta^{2}\right) e^{-K \eta} \\
& +\left(\frac{C_{1}+C_{3}+C_{5}}{K}+C_{3} \eta+C_{4} \eta^{2}\right) e^{-2 K \eta} \\
& +\left(C_{5} \eta+C_{6} \eta^{2}\right) e^{-3 K \eta} .
\end{aligned}
$$


The first-order approximate solution given by (26) is obtained from (35) and (42):

$$
\begin{aligned}
\left(A_{1}\right) \bar{f}\left(\eta, C_{i}\right)= & R+\frac{1}{K}+\left(-\frac{1+C_{1}+C_{3}+C_{5}}{K}\right. \\
& \left.+C_{1} \eta+C_{2} \eta^{2}\right) e^{-K \eta} \\
& +\left(\frac{C_{1}+C_{3}+C_{5}}{K}+C_{3} \eta+C_{4} \eta^{2}\right) e^{-2 K \eta} \\
& +\left(C_{5} \eta+C_{6} \eta^{2}\right) e^{-3 K \eta} .
\end{aligned}
$$

In this way, we can find other solutions:

$$
\begin{aligned}
\left(A_{2}\right) \bar{f}\left(\eta, C_{i}\right)= & \frac{1}{K}+\left(2 R-\frac{1+C_{1}+C_{3}+C_{5}}{K}\right. \\
& \left.+C_{1} \eta+C_{2} \eta^{2}\right) e^{-K \eta} \\
& +\left(\frac{C_{1}+C_{3}+C_{5}}{K}-R+C_{3} \eta\right. \\
& \left.+C_{4} \eta^{2}\right) e^{-2 K \eta}+\left(C_{5} \eta+C_{6} \eta^{2}\right) e^{-3 K \eta}, \\
\left(A_{3}\right) \bar{f}\left(\eta, C_{i}\right)= & R+\frac{1}{K^{2}}+\left(-\frac{C_{1}+C_{3}+C_{5}}{2 K}+\frac{K-3}{2 K^{2}}\right. \\
& +\left(C_{3} \eta+C_{4} \eta^{2}\right) e^{-2 K \eta} \\
& +\left(\frac{1-K}{2 K^{2}}+\frac{C_{1}+C_{3}+C_{5}}{2 K}+C_{5} \eta+C_{6} \eta^{2}\right) \\
& \times e^{-3 K \eta}, \quad
\end{aligned}
$$

$\left(A_{4}\right) \bar{f}\left(\eta, C_{i}\right)=R+\frac{1}{K}+\left(-\frac{1}{K}+C_{1} \eta^{2}+C_{2} \eta^{3}\right.$

$$
\left.+C_{3} \eta^{4}\right) e^{-K \eta}
$$

$$
+\left(C_{4} \eta^{2}+C_{5} \eta^{3}+C_{6} \eta^{4}\right) e^{-2 K \eta}
$$

$\left(A_{5}\right) \bar{f}\left(\eta, C_{i}\right)=R+\frac{1}{K}+\left(-\frac{1}{K}+C_{1} \eta+C_{2} \eta^{2}+C_{3} \eta^{3}\right.$

$$
\begin{gathered}
\left.+C_{4} \eta^{4}\right) e^{-K \eta} \\
+\left(-C_{1} \eta+C_{5} \eta^{2}+C_{6} \eta^{3}\right) e^{-2 K \eta},
\end{gathered}
$$

$\left(A_{6}\right) \bar{f}\left(\eta, C_{i}\right)=R+\frac{1}{K^{2}}+\left(-\frac{1}{K^{2}}+\left(1-\frac{1}{K}\right) \eta+C_{1} \eta^{2}\right.$

$$
\begin{array}{r}
\left.+C_{2} \eta^{3}+C_{3} \eta^{4}\right) e^{-K \eta} \\
+\left(C_{4} \eta^{2}+C_{5} \eta^{3}+C_{6} \eta^{4}\right) e^{-2 K \eta}
\end{array}
$$

$$
\begin{aligned}
& \left(A_{7}\right) \bar{f}\left(\eta, C_{i}\right)=R+\frac{1-e^{-K \eta}}{K}+\left(C_{1} \eta^{2}+C_{2} \eta^{3}\right. \\
& \left.+C_{3} \eta^{4}\right) e^{-2 K \eta} \\
& +\left(C_{4} \eta^{2}+C_{5} \eta^{3}+C_{6} \eta^{4}\right) e^{-3 K \eta} \\
& \left(A_{8}\right) \bar{f}\left(\eta, C_{i}\right)=R+\frac{1}{K}\left(1-e^{-K \eta}\right)+\left(3 C_{1} \eta+C_{2} \eta^{2}\right. \\
& \left.+C_{3} \eta^{3}\right) e^{-2 K \eta} \\
& +\left(-2 C_{1} \eta+C_{4} \eta^{2}+C_{5} \eta^{3}+C_{6} \eta^{4}\right) e^{-3 K \eta} \\
& \left(A_{9}\right) \bar{f}\left(\eta, C_{i}\right)=R+\frac{1}{K^{2}}\left(1-e^{-K \eta}\right)+\left[\left(1-\frac{1}{K}\right) \eta+C_{1} \eta^{2}\right. \\
& \left.+C_{2} \eta^{3}+C_{3} \eta^{4}\right] \\
& \times e^{-2 K \eta}+\left(C_{4} \eta^{2}+C_{5} \eta^{3}+C_{6} \eta^{4}\right) e^{-3 K \eta}, \\
& \left(A_{10}\right) \bar{f}\left(\eta, C_{i}\right)=R+\frac{1}{K}+\left(-\frac{1}{K}+C_{1} \eta^{2}+C_{2} \eta^{3}+C_{3} \eta^{4}\right) \\
& \times e^{-K \eta}+\left(C_{4} \eta^{2}+C_{5} \eta^{3}+C_{6} \eta^{4}\right) e^{-3 K \eta}, \\
& \left(A_{11}\right) \bar{f}\left(\eta, C_{i}\right)=R+\frac{1}{K}+\left(-\frac{1}{K}+C_{1} \eta+C_{2} \eta^{2}\right) e^{-K \eta} \\
& +\left(C_{3} \eta+C_{4} \eta^{2}\right) e^{-2 K \eta}+\left(C_{5} \eta+C_{6} \eta^{2}\right) \\
& \times e^{-3 K \eta}+\left[\left(-C_{1}-C_{3}-C_{5}\right) \eta+C_{7} \eta^{2}\right] \\
& \times e^{-4 K \eta} \\
& \left(A_{12}\right) \bar{f}\left(\eta, C_{i}\right)=R+\frac{1}{K}+\left(-\frac{1}{K}+C_{1} \eta^{2}\right) e^{-K \eta} \\
& +\left(C_{2} \eta^{2}+C_{3} \eta^{3}\right) e^{-2 K \eta} \\
& +\left(C_{4} \eta^{2}+C_{5} \eta^{3}+C_{6} \eta^{4}\right) e^{-3 K \eta} \text {. }
\end{aligned}
$$

It is clear that we can obtain many other solutions.

\section{Numerical Results}

We illustrate the accuracy of our procedure for different values of the coefficients $R, \beta$, and $M$. Also, we represent graphically the behavior of the functions $f$ and $f^{\prime}$ and we compare results obtained through our procedure with numerical results.

(1) First, we consider $\beta=0.5, M=0.75$, and $R=$ 0.25 which corresponds to suction velocity. The optimal convergence-control parameters are determined by means of the least-square method and are as follows:

$$
\begin{aligned}
\left(A_{1}\right) K & =1.7223999348, \quad C_{1}=0.0203632630, \\
C_{2} & =-0.0003551855, \quad C_{3}=-0.0046442857, \\
C_{4} & =0.0021151819, \quad C_{5}=-0.0040337933, \\
C_{6} & =-0.0030441083,
\end{aligned}
$$




$$
\begin{aligned}
& \left(A_{2}\right) K=1.2039668671, \quad C_{1}=0.0484595533, \\
& C_{2}=-0.0035879577, \quad C_{3}=-0.1933189832, \\
& C_{4}=-0.0613273780, \quad C_{5}=-0.0321447762 \text {, } \\
& C_{6}=-0.0048370121
\end{aligned}
$$

and so on.

The first-order approximate solutions obtained by means OHAM, for the above cases $\left(A_{1}\right)-\left(A_{12}\right)$, become as follows:

$$
\begin{aligned}
\left(A_{1}\right) \bar{f}(\eta)= & 0.8305852518+(-0.5873694973 \\
& +0.0203632630 \eta \\
& \left.-0.0003551855 \eta^{2}\right) \\
& \times e^{-1.7223999348 \eta} \\
& +(0.0067842454-0.0046442857 \eta \\
& \left.+0.0021151819 \eta^{2}\right) e^{-3.4447998696 \eta} \\
& +\left(-0.0040337933 \eta-0.0030441083 \eta^{2}\right) \\
& \times e^{-5.1671998044 \eta}
\end{aligned}
$$

$$
\begin{aligned}
\left(A_{2}\right) \bar{f}(\eta)=0.8305876410+( & -0.1835701350 \\
+ & 0.0484595533 \eta \\
& \left.-0.0035879577 \eta^{2}\right) \\
\times e^{-1.2039668671 \eta}+( & -0.3970175060 \\
& -0.1933189832 \eta \\
& \left.-0.0613273780 \eta^{2}\right) \\
\times e^{-2.4079337342 \eta}+( & -0.0321447762 \eta \\
& \times e^{-3.6119006013 \eta},
\end{aligned}
$$

$$
\begin{aligned}
\left(A_{3}\right) \bar{f}(\eta)=0.8305880207+( & -0.3420493544 \\
+ & 0.0892570980 \eta \\
& \left.-0.0066950727 \eta^{2}\right) \\
\times e^{-1.3123992230 \eta}+( & -0.2899683613 \eta \\
& \left.-0.0166914996 \eta^{2}\right) \\
\times e^{-2.6247984460 \eta}+( & -0.2385386662 \\
& -0.1873679245 \eta \\
& \times e^{-3.9371976690 \eta},
\end{aligned}
$$

$\left(A_{4}\right) \bar{f}(\eta)=0.8305846668+(-0.5805846668$

$$
\begin{aligned}
& +0.0113141332 \eta^{2} \\
& -0.0026254854 \eta^{3} \\
& \left.+0.0002157623 \eta^{4}\right)
\end{aligned}
$$

$$
\begin{aligned}
& \times e^{-1.7224016705 \eta} \\
& +\left(0.0194551449 \eta^{2}+0.0055884706 \eta^{3}\right. \\
& \left.\quad+0.0021434013 \eta^{4}\right) e^{-3.4448033410 \eta}
\end{aligned}
$$

$$
\begin{aligned}
\left(A_{5}\right) \bar{f}(\eta)=0.8305850786+( & -0.5805850786 \\
& +0.0129138333 \eta \\
& +0.0029826874 \eta^{2} \\
& -0.0007022807 \eta^{3} \\
& \left.+0.0000575450 \eta^{4}\right) \\
& \times e^{-1.7224004488 \eta \quad} \\
+ & \\
& -0.0129138333 \eta+0.0055128320 \eta^{2} \\
& \left.-0.009540905 \eta^{3}\right) e^{-3.4448008976 \eta}
\end{aligned}
$$

$$
\begin{aligned}
\left(A_{6}\right) \bar{f}(\eta)=0.8305896186+( & -0.5805896186 \\
& +0.2380356841 \eta \\
& -0.0458010823 \eta^{2} \\
& +0.0050361161 \eta^{3} \\
& \left.-0.0002634636 \eta^{4}\right)
\end{aligned}
$$

$$
\times e^{-1.3123974169 \eta}
$$$$
+\left(0.0276184306 \eta^{2}-0.0042554588 \eta^{3}\right.
$$$$
\left.+0.0040951218 \eta^{4}\right) e^{-2.6247948339 \eta},
$$

$\left(A_{7}\right) \bar{f}(\eta)=0.25+0.5805981621\left(1-e^{-1.7223616352 \eta}\right)$

$$
\begin{gathered}
+\left(3.7465003467 \eta^{2}-2.5918747904 \eta^{3}\right. \\
\left.+0.5688873710 \eta^{4}\right) e^{-3.4447232705 \eta} \\
+\left(-3.7216397470 \eta^{2}-3.7194132780 \eta^{3}\right. \\
\left.-1.7743745762 \eta^{4}\right) e^{-5.1670849057 \eta}
\end{gathered}
$$




$$
\begin{aligned}
\left(A_{8}\right) \bar{f}(\eta)= & 0.25+0.5806004947\left(1-e^{-1.7223547156 \eta}\right) \\
+ & \left(28.6559933963 \eta-17.1574454736 \eta^{2}\right. \\
& \left.+3.0755892307 \eta^{3}\right) e^{-3.4447094313 \eta} \\
+ & \left(-28.6559933963 \eta-32.1751588943 \eta^{2}\right. \\
& \left.\quad-15.8637665758 \eta^{3}-4.4540759880 \eta^{4}\right) \\
& \times e^{-5.1670641469 \eta}
\end{aligned}
$$

$$
\begin{aligned}
\left(A_{9}\right) \bar{f}(\eta)= & 0.25+0.5807600837\left(1-e^{-1.3122047950 \eta}\right) \\
+ & \left(0.2379238333 \eta+14.3490983671 \eta^{2}\right. \\
& \left.\quad-7.4936309491 \eta^{3}+1.33465899985 \eta^{4}\right) \\
& \times e^{-2.6244095900 \eta} \\
+ & \left(-14.0803697555 \eta^{2}-10.6121821644 \eta^{3}\right. \\
& \left.\quad-3.7974679259 \eta^{4}\right) e^{-3.9366143850 \eta}
\end{aligned}
$$

$\left(A_{10}\right) \bar{f}(\eta)=0.8305805525+(-0.5805805525$

$$
\begin{aligned}
& +0.0163027398 \eta^{2} \\
& -0.0053622456 \eta^{3} \\
& \left.+0.0006113624 \eta^{4}\right)
\end{aligned}
$$

$$
\begin{aligned}
& \times e^{-1.7224138763 \eta} \\
& +\left(0.0153758771 \eta^{2}+0.0170806180 \eta^{3}\right. \\
& \left.+0.0369073209 \eta^{4}\right) e^{-5.1672416289 \eta}, \\
& \left(A_{11}\right) \bar{f}(\eta)=0.8305850069+(-0.5805850069 \\
& +0.0167016213 \eta \\
& \left.+0.0001742425 \eta^{2}\right) \\
& \times e^{-1.7224006615 \eta} \\
& +\left(-0.0115884531 \eta-0.0052672814 \eta^{2}\right) \\
& \times e^{-3.4448013230 \eta}+(-0.0193966267 \eta \\
& \left.+0.0114385136 \eta^{2}\right) \\
& \times e^{-5.1672019845 \eta}+(0.0142834585 \eta \\
& \left.+0.0114385136 \eta^{2}\right) \\
& \times e^{-6.8896026460 \eta},
\end{aligned}
$$

$$
\begin{aligned}
\left(A_{12}\right) \bar{f}(\eta)= & 0.8305751642+(-0.5805751642 \\
& \left.+0.0012101182 \eta^{3}\right) \\
& \times e^{-1.7224298620 \eta} \\
& +\left(-0.4645923536 \eta^{2}+0.2718146543 \eta^{3}\right) \\
& \times e^{-3.4448597240 \eta} \\
& +\left(0.4983783090 \eta^{2}+0.5722116290 \eta^{3}\right. \\
& \left.\quad+0.4013983403 \eta^{4}\right) e^{-5.1672895860 \eta}
\end{aligned}
$$

(2) In the last case, we consider $\beta=0.5, M=0.75$, and $R=-2$ for injection velocity. The optimal convergencecontrol parameters are as follows:

$$
\begin{aligned}
& \left(A_{1}\right) K=3.4039784470, \quad C_{1}=-0.0073489093, \\
& C_{2}=-0.0000300879, \quad C_{3}=0.0019346837, \\
& C_{4}=-0.0028434644, \quad C_{5}=0.0004870868, \\
& C_{6}=0.0008838343 \text {, } \\
& \left(A_{2}\right) K=0.3235777833, \quad C_{1}=193.0767116481 \text {, } \\
& C_{2}=-9.2315115218, \quad C_{3}=269.5805006985, \\
& C_{4}=10.4423160474, \quad C_{5}=-36.4073561981, \\
& C_{6}=-8.0939438081
\end{aligned}
$$

and so on.

The first-order approximate solutions can be written for only three cases in the following forms:

$$
\begin{aligned}
\left(A_{1}\right) \bar{f}(\eta)= & -1.7062261070+(-0.2923264282 \\
& -0.0073489093 \eta \\
& \left.-0.0000300879 \eta^{2}\right) \\
& +(-0.0014474647+0.0019346837 \eta \\
& \left.\quad-0.0028434644 \eta^{2}\right) e^{-6.8079568940 \eta} \\
& +\left(0.0004870868 \eta+0.0008838343 \eta^{2}\right) \\
& \times e^{-10.2119353411 \eta}
\end{aligned}
$$




$$
\begin{aligned}
\left(A_{4}\right) \bar{f}(\eta)= & -1.7062260957+(-0.2937739042 \\
& -0.0079483534 \eta^{2} \\
& +0.0033559204 \eta^{3} \\
& \left.-0.0005109100 \eta^{4}\right) \\
& \times e^{-3.4039783163 \eta} \quad \\
& +\left(-0.0123589233 \eta^{2}-0.0091292547 \eta^{3}\right. \\
& \times e^{-6.8079566326 \eta},
\end{aligned}
$$

$$
\begin{aligned}
\left(A_{11}\right) \bar{f}(\eta)= & -1.7062260959+(-0.2937739040 \\
& -0.0058506315 \eta \\
& \left.\times e^{-3.4039783189 \eta} \quad-0004400135 \eta^{2}\right) \\
& +\left(0.0043294258 \eta+0.0042247893 \eta^{2}\right) \\
& \times e^{-6.8079566378 \eta} \quad \\
& +\left(0.0072464953 \eta-0.0092559326 \eta^{2}\right) \\
& \times e^{-10.2119349567 \eta} \\
& +\left(-0.0057252895 \eta-0.0092559326 \eta^{2}\right) \\
& \times e^{-13.6159132756 \eta} .
\end{aligned}
$$

In Figures 1 and 2, a comparison between the firstorder approximate solutions $\bar{f}(\eta)$ and $\bar{f}^{\prime}(\eta)$, respectively, for the suction velocity in the case $\left(A_{1}\right)$ and numerical results is plotted, while, in Figures 3 and 4, a comparison between the first-order approximate solutions $\bar{f}(\eta)$ and $\bar{f}^{\prime}(\eta)$, respectively, for the injection velocity in the case $\left(A_{1}\right)$ is plotted.

In Tables 1, 2, 3, 4, and 5, we present a comparison between the first-order approximate solutions (46)-(62), respectively, with numerical results for some values of variable $\eta$.

In Tables 6, 7, and 8, we present the relative error between the first-order approximate solutions (46)-(62) and numerical results; $\varepsilon=\left|\bar{f}_{\text {OHAM }}(\eta)-f_{\text {numerical }}(\eta)\right|$ for some values of variable $\eta$.

In Tables 9 and 10, we present a comparison between the skin friction coefficient $\bar{F}^{\prime \prime}(0)$ and the value of $\bar{F}(\infty)$ by means of OHAM and numerical integrations. All these comparisons are found to be in very good agreement with the first-order approximate solution obtained by OHAM.

It can be seen from the above Tables that the solutions obtained by the proposed procedure are nearly identical

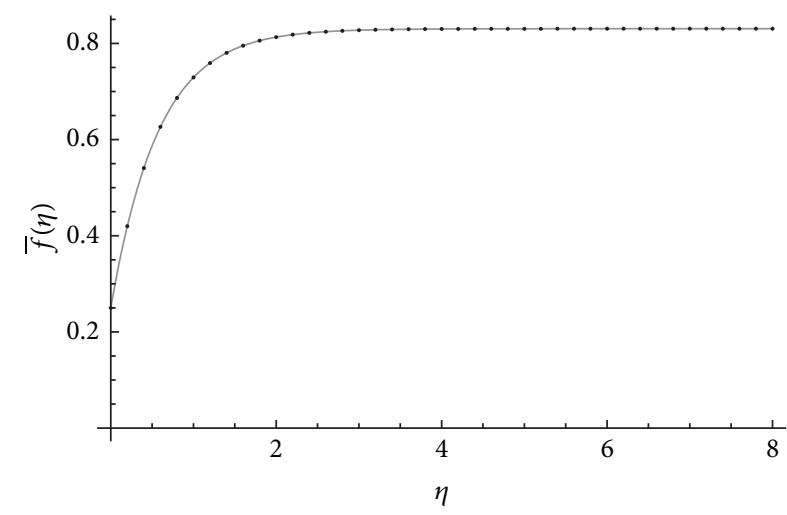

FIGURE 1: Comparison between the approximate solution (46) and numerical solution in the following cases: $\beta=0.5, M=0.75$, and $R=0.25$-numerical solution; ........ approximate solution.

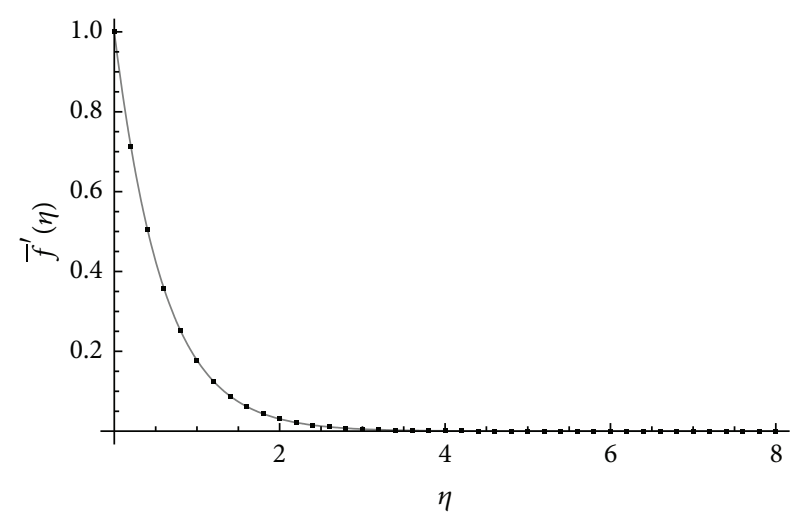

Figure 2: Comparison between the derivative of the first-order approximate solution of (46) and numerical solution in the following cases: $\beta=0.5, M=0.75$, and $R=0.25$-numerical solution; $\ldots \ldots$ approximate solution.

with the numerical solutions obtained using a fourth-order Runge-Kutta method in combination with the shooting method.

Also, we note that one can get a lot of other solutions by means of the initial approximation given by (36).

Some statistical tests are necessary to sustain some asymptotic properties. In fact, two statistical tests are more important: test of homoscedasticity and test of autocorrelations. We compute the Durbin-Watson test for autocorrelation and Bartlett test for homoscedasticity:

$$
\begin{gathered}
F=\frac{\sum_{i=1}^{[n / 2]} \varepsilon_{i}^{2}}{\sum_{i=[n / 2]+1}^{n} \varepsilon_{i}^{2}} \quad \text { (Barlett test), } \\
d=\frac{\sum_{i=1}^{2 n}\left(\varepsilon_{i}-\varepsilon_{i-1}\right)^{2}}{\sum_{i=1}^{n} \varepsilon_{i}^{2}} \quad \text { (Durbin-Wattson test). }
\end{gathered}
$$

The representative limits for Barlett test are $F_{\text {inf }}=$ $0.000162 ; F_{\text {sup }}=8.6831168141$ for confidence lavel $0.99 \%$ (the errors $\varepsilon_{i}=\bar{f}_{\text {OHAM }}\left(\eta_{i}\right)-f_{\text {numerical }}\left(\eta_{i}\right), i=1,2, \ldots, n$ pass the Barlett test if $\left.F_{\text {inf }}<F<F_{\text {sup }}\right)$. This test assure that errors 
TABLE 1: Comparison of the first-order approximate solution $\bar{f}$ obtained by OHAM with numerical results.

\begin{tabular}{lcccr}
\hline$\eta$ & $\bar{f}_{\text {OHAM }}(\eta)$ & $\bar{f}_{\text {OHAM }}(\eta)$ & $\bar{f}_{\text {OHAM }}(\eta)$ & $(48)$ \\
& $(46)$ & $(47)$ & 0.25 & $f_{\text {numerical }}(\eta)$ \\
\hline 0 & 0.25 & 0.25 & 0.6867555458 & 0.25 \\
$8 / 5$ & 0.6867557456 & 0.6867558261 & 0.7952835769 & 0.6867557531 \\
$12 / 5$ & 0.7952831540 & 0.7952832405 & 0.82192586666 & 0.7952831536 \\
$16 / 5$ & 0.8219262765 & 0.8219262098 & 0.8284615570 & 0.8219262711 \\
4 & 0.8284614196 & 0.8284613427 & 0.8300647355 & 0.8300642946131 \\
$24 / 5$ & 0.8300643020 & 0.8300645259 & 0.8304573214 & 0.8304574344 \\
$28 / 5$ & 0.8304574558 & 0.8304574869 & 0.8305535912 & 0.8305538603 \\
$32 / 5$ & 0.8305538969 & 0.8305537376 & 0.8305777588 & 0.8305775112 \\
$36 / 5$ & 0.8305775573 & 0.8305776155 & 0.8305843430 & 0.8305833139 \\
8 & 0.8305833631 & 0.8305839424 & 0.8305864617 & 0.8305847388 \\
\hline
\end{tabular}

TABLE 2: Comparison of the first-order approximate solution $\bar{f}$ obtained by OHAM with numerical results.

\begin{tabular}{lcccr}
\hline$\eta$ & $\bar{f}_{\text {OHAM }}(\eta)$ & $\bar{f}_{\text {OHAM }}(\eta)$ & $\bar{f}_{\text {OHAM }}(\eta)$ & $(51)$ \\
\hline 0 & $(49)$ & $(50)$ & 0.25 & 0.25 \\
$4 / 5$ & 0.25 & 0.25 & 0.6867559421 & 0.6867557531 \\
$8 / 5$ & 0.6867559559 & 0.6867558676 & 0.7952832693 & 0.7952831536 \\
$12 / 5$ & 0.7952829566 & 0.7952830577 & 0.8219262460 & 0.8219262711 \\
$16 / 5$ & 0.8219264714 & 0.8219263597 & 0.8284612078 & 0.8284614131 \\
4 & 0.8284612774 & 0.8284613647 & 0.8300646204 & 0.8300642946 \\
$24 / 5$ & 0.8300641916 & 0.8300642631 & 0.8304575594 & 0.8304574344 \\
$28 / 5$ & 0.8304575489 & 0.8304574866 & 0.8305536600 & 0.8305538603 \\
$32 / 5$ & 0.8305539626 & 0.8305539177 & 0.8305775904 & 0.8305775112 \\
$36 / 5$ & 0.8305774335 & 0.8305775190 & 0.8305842233 & 0.8305833139 \\
8 & 0.8305830455 & 0.8305832668 & 0.8305866484 & 0.8305847388 \\
\hline
\end{tabular}

TABLE 3: Comparison of the first-order approximate solution $\bar{f}$ obtained by OHAM with numerical results.

\begin{tabular}{lcccc}
\hline$\eta$ & $\bar{f}_{\text {OHAM }}(\eta)$ & $\bar{f}_{\text {OHAM }}(\eta)$ & $\bar{f}_{\text {OHAM }}(\eta)$ & $(54)$ \\
& $(52)$ & $(53)$ & 0.25 & 0.25 \\
$4 / 5$ & 0.25 & 0.25 & 0.6866887026 & 0.6867557531 \\
$8 / 5$ & 0.6867604947 & 0.6867642071 & 0.7952830603 & 0.7952831536 \\
$12 / 5$ & 0.7952711920 & 0.7952698391 & 0.8219258072 & 0.8219262711 \\
$16 / 5$ & 0.8219400081 & 0.8219414386 & 0.8285164738 & 0.8284614131 \\
4 & 0.8284434011 & 0.8284360789 & 0.8299931937 & 0.8300642946 \\
$24 / 5$ & 0.8300472216 & 0.8300445299 & 0.8303854806 & 0.8304574344 \\
$28 / 5$ & 0.8304557614 & 0.8304567567 & 0.8305702204 & 0.8305538603 \\
$32 / 5$ & 0.8305615064 & 0.8305635876 & 0.8306729872 & 0.8305775112 \\
$36 / 5$ & 0.8305888008 & 0.8305910952 & 0.8307238267 & 0.8305833139 \\
8 & 0.8305957861 & 0.8305981136 & 0.8307459962 & \\
\hline
\end{tabular}


TABLE 4: Comparison of the first-order approximate solution $\bar{f}$ obtained by OHAM with numerical results.

\begin{tabular}{lcccr}
\hline$\eta$ & $\bar{f}_{\text {OHAM }}(\eta)$ & $\bar{f}_{\text {OHAM }}(\eta)$ & $\bar{f}_{\text {OHAM }}(\eta)$ & $(57)$ \\
$(55)$ & $(56)$ & 0.25 & 0.25 \\
$4 / 5$ & 0.25 & 0.25 & 0.6867565108 & 0.6867557531 \\
$8 / 5$ & 0.6867577703 & 0.6867556858 & 0.7952904892 & 0.7952831536 \\
$12 / 5$ & 0.7952835590 & 0.7952833690 & 0.8219182801 & 0.8219262711 \\
$16 / 5$ & 0.8219267050 & 0.8219260684 & 0.8284624519 & 0.8284614131 \\
4 & 0.8284593204 & 0.8284614530 & 0.8300733641 & 0.8300642946 \\
$24 / 5$ & 0.8300649028 & 0.8300645204 & 0.8304617717 & 0.8304574344 \\
$28 / 5$ & 0.8304590162 & 0.8304575585 & 0.8305514864 & 0.8305538603 \\
$32 / 5$ & 0.8305540337 & 0.8305538374 & 0.8305708821 & 0.8305775112 \\
$36 / 5$ & 0.8305757755 & 0.8305773944 & 0.8305746354 & 0.8305833139 \\
8 & 0.8305801662 & 0.8305831505 & 0.8305752047 & 0.8305847388 \\
\hline
\end{tabular}

TABLE 5: Comparison of the first-order approximate solution $\bar{f}$ obtained by OHAM with numerical results.

\begin{tabular}{lcccr}
\hline$\eta$ & $\bar{f}_{\text {OHAM }}(\eta)$ & $\bar{f}_{\text {OHAM }}(\eta)$ & $\bar{f}_{\text {OHAM }}(\eta)$ & $(62)$ \\
\hline 0 & $(60)$ & $(61)$ & -2 & $f_{\text {numerical }}(\eta)$ \\
$4 / 5$ & -2 & -2 & -1.7258163629 & -2 \\
$8 / 5$ & -1.7258163099 & -1.7258162625 & -1.7075377264 & -1.7258163121 \\
$12 / 5$ & -1.7075377409 & -1.7075377235 & -1.7063139675 & -1.7075377364 \\
$16 / 5$ & -1.7063139221 & -1.7063139346 & -1.7062319899 & -1.7062319847 \\
4 & -1.7062319851 & -1.7062320229 & -1.7062264918 & -1.7062264984 \\
$24 / 5$ & -1.7062265004 & -1.7062265071 & -1.7062261225 & -1.7062261309 \\
$28 / 5$ & -1.7062261333 & -1.7062261259 & -1.7062260977 & -1.7062261063 \\
$32 / 5$ & -1.7062261087 & -1.7062260981 & -1.7062260960 & -1.7062261046 \\
$36 / 5$ & -1.7062261071 & -1.7062260959 & -1.7062260959 & -1.7062261044 \\
8 & -1.7062261070 & -1.7062260957 & -1.7062260959 & -1.7062261044 \\
\hline
\end{tabular}

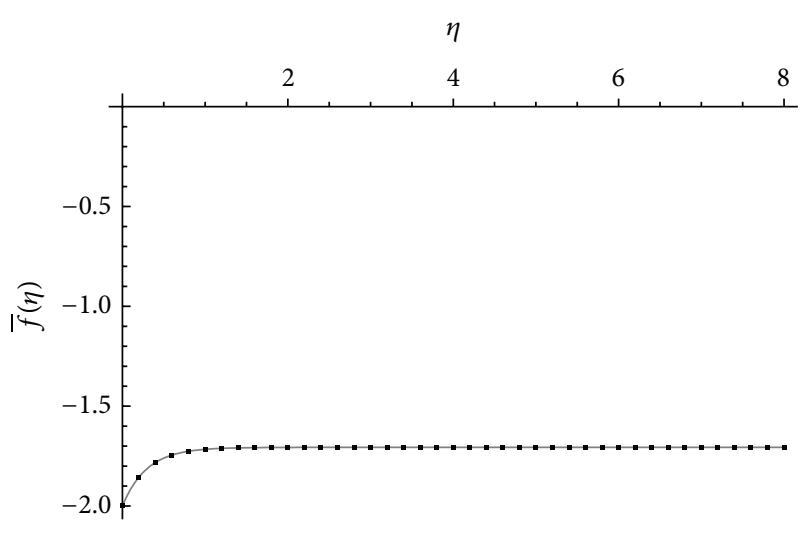

FIGURE 3: Comparison between the approximate solution (60) and numerical solution in the following cases: $\beta=0.5, M=0.75$, and $R=-2-$ numerical solution; ......... approximate solution.

have the constant variance and possible to control the analytic approximate solutions for any real interval.

The representative limits for Durbin-Wattson test are $d_{\text {inf }}=1.36 ; d_{\text {sup }}=2.92$ for confidence lavel $0.99 \%$ (again,

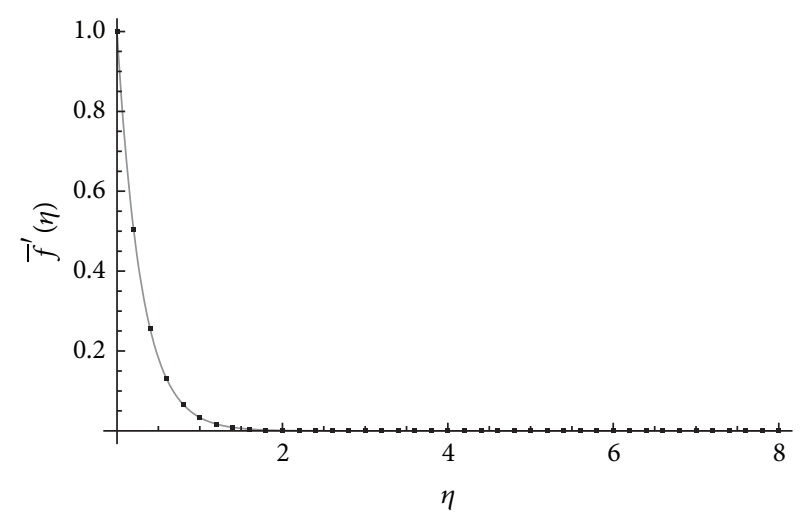

Figure 4: Comparison between the derivative of the first-order approximate solution of (60) and numerical solution in the following cases: $\beta=0.5, M=0.75$, and $R=-2$-numerical solution; ........ approximate solution.

the all errors $\varepsilon_{i}=\bar{f}_{\text {OHAM }}\left(\eta_{i}\right)-f_{\text {numerical }}\left(\eta_{i}\right), i=1,2, \ldots, n$ pass the Durbin-Wattson test, that is, $\left.d_{\text {inf }}<d<d_{\text {sup }}\right)$. This test explain no correlation between errors and this means that 
TABLE 6: Comparison of the first-order approximate solution $\bar{f}$ obtained by OHAM with numerical results.

\begin{tabular}{lccccc}
\hline$\eta$ & $\varepsilon$ & $\varepsilon$ & $\varepsilon$ & $\varepsilon$ & $\varepsilon$ \\
& $(46)$ & $(47)$ & $(48)$ & $(49)$ & $(50)$ \\
\hline 0 & $1.1 \cdot 10^{-16}$ & $1.1 \cdot 10^{-16}$ & 0 & 0 & 0 \\
$4 / 5$ & $7.8 \cdot 10^{-9}$ & $7.2 \cdot 10^{-8}$ & $2.0 \cdot 10^{-7}$ & $2.0 \cdot 10^{-7}$ & $1.1 \cdot 10^{-7}$ \\
$8 / 5$ & $9.0 \cdot 10^{-10}$ & $8.5 \cdot 10^{-8}$ & $4.2 \cdot 10^{-7}$ & $1.9 \cdot 10^{-7}$ & $9.7 \cdot 10^{-8}$ \\
$12 / 5$ & $1.9 \cdot 10^{-9}$ & $6.4 \cdot 10^{-8}$ & $4.0 \cdot 10^{-7}$ & $1.9 \cdot 10^{-7}$ & $8.5 \cdot 10^{-8}$ \\
$16 / 5$ & $4.0 \cdot 10^{-10}$ & $7.7 \cdot 10^{-8}$ & $1.4 \cdot 10^{-7}$ & $1.4 \cdot 10^{-7}$ & $5.5 \cdot 10^{-8}$ \\
4 & $4.7 \cdot 10^{-9}$ & $2.1 \cdot 10^{-7}$ & $4.4 \cdot 10^{-7}$ & $1.1 \cdot 10^{-7}$ & $4.3 \cdot 10^{-8}$ \\
$24 / 5$ & $1.2 \cdot 10^{-9}$ & $3.2 \cdot 10^{-8}$ & $1.1 \cdot 10^{-7}$ & $9.4 \cdot 10^{-8}$ & $3.1 \cdot 10^{-8}$ \\
$28 / 5$ & $4.5 \cdot 10^{-9}$ & $1.5 \cdot 10^{-7}$ & $2.6 \cdot 10^{-7}$ & $7.0 \cdot 10^{-8}$ & $2.5 \cdot 10^{-8}$ \\
$32 / 5$ & $3.5 \cdot 10^{-9}$ & $5.4 \cdot 10^{-8}$ & $2.4 \cdot 10^{-7}$ & $1.2 \cdot 10^{-7}$ & $4.1 \cdot 10^{-8}$ \\
$36 / 5$ & $2.6 \cdot 10^{-8}$ & $5.5 \cdot 10^{-7}$ & $1.0 \cdot 10^{-6}$ & $3.4 \cdot 10^{-7}$ & $1.2 \cdot 10^{-7}$ \\
8 & $6.4 \cdot 10^{-8}$ & $1.0 \cdot 10^{-6}$ & $1.7 \cdot 10^{-6}$ & $5.1 \cdot 10^{-7}$ & $1.9 \cdot 10^{-7}$ \\
\hline
\end{tabular}

TABLE 7: Comparison of the first-order approximate solution $\bar{f}$ obtained by OHAM with numerical results.

\begin{tabular}{lccccc}
\hline$\eta$ & $\varepsilon$ & $\varepsilon$ & $\varepsilon$ & $\varepsilon$ & $\varepsilon$ \\
& $(51)$ & $(52)$ & $(53)$ & $(54)$ & $(55)$ \\
\hline 0 & 0 & 0 & 0 & 0 & 0 \\
$4 / 5$ & $1.8 \cdot 10^{-7}$ & $4.7 \cdot 10^{-6}$ & $8.4 \cdot 10^{-6}$ & $6.7 \cdot 10^{-5}$ & $2.0 \cdot 10^{-6}$ \\
$8 / 5$ & $1.1 \cdot 10^{-7}$ & $1.1 \cdot 10^{-5}$ & $1.3 \cdot 10^{-5}$ & $9.4 \cdot 10^{-8}$ & $4.0 \cdot 10^{-7}$ \\
$12 / 5$ & $2.8 \cdot 10^{-8}$ & $1.3 \cdot 10^{-5}$ & $1.5 \cdot 10^{-5}$ & $4.6 \cdot 10^{-7}$ & $4.3 \cdot 10^{-7}$ \\
$16 / 5$ & $2.1 \cdot 10^{-7}$ & $1.8 \cdot 10^{-5}$ & $2.5 \cdot 10^{-5}$ & $5.5 \cdot 10^{-5}$ & $2.0 \cdot 10^{-6}$ \\
4 & $3.1 \cdot 10^{-7}$ & $1.7 \cdot 10^{-5}$ & $1.9 \cdot 10^{-5}$ & $7.1 \cdot 10^{-5}$ & $5.9 \cdot 10^{-7}$ \\
$24 / 5$ & $1.0 \cdot 10^{-7}$ & $1.6 \cdot 10^{-6}$ & $6.9 \cdot 10^{-7}$ & $7.1 \cdot 10^{-5}$ & $1.5 \cdot 10^{-6}$ \\
$28 / 5$ & $2.3 \cdot 10^{-7}$ & $7.6 \cdot 10^{-6}$ & $9.6 \cdot 10^{-6}$ & $1.6 \cdot 10^{-5}$ & $1.4 \cdot 10^{-7}$ \\
$32 / 5$ & $2.9 \cdot 10^{-8}$ & $1.1 \cdot 10^{-5}$ & $1.3 \cdot 10^{-5}$ & $9.5 \cdot 10^{-5}$ & $1.7 \cdot 10^{-6}$ \\
$36 / 5$ & $8.3 \cdot 10^{-7}$ & $1.2 \cdot 10^{-5}$ & $1.4 \cdot 10^{-5}$ & $1.4 \cdot 10^{-5}$ & $3.2 \cdot 10^{-6}$ \\
8 & $1.7 \cdot 10^{-6}$ & $1.2 \cdot 10^{-5}$ & $1.5 \cdot 10^{-5}$ & $1.6 \cdot 10^{-5}$ & $4.0 \cdot 10^{-6}$ \\
\hline
\end{tabular}

TABLE 8: Comparison of the first-order approximate solution $\bar{f}$ obtained by OHAM with numerical results.

\begin{tabular}{lccccc}
\hline$\eta$ & $\varepsilon$ & $\varepsilon$ & $\varepsilon$ & $\varepsilon$ & $\varepsilon$ \\
& $(56)$ & $(57)$ & $(60)$ & $(61)$ & $(62)$ \\
\hline 0 & 0 & 0 & 0 & 0 & 0 \\
$4 / 5$ & $6.7 \cdot 10^{-8}$ & $7.5 \cdot 10^{-7}$ & $2.4 \cdot 10^{-9}$ & $4.9 \cdot 10^{-8}$ & $5.0 \cdot 10^{-8}$ \\
$8 / 5$ & $2.1 \cdot 10^{-7}$ & $7.3 \cdot 10^{-6}$ & $3.8 \cdot 10^{-9}$ & $1.3 \cdot 10^{-8}$ & $9.9 \cdot 10^{-9}$ \\
$12 / 5$ & $2.0 \cdot 10^{-7}$ & $7.9 \cdot 10^{-6}$ & $4.0 \cdot 10^{-9}$ & $8.5 \cdot 10^{-9}$ & $4.2 \cdot 10^{-8}$ \\
$16 / 5$ & $3.2 \cdot 10^{-8}$ & $1.0 \cdot 10^{-6}$ & $8.8 \cdot 10^{-10}$ & $3.6 \cdot 10^{-8}$ & $5.1 \cdot 10^{-9}$ \\
4 & $2.1 \cdot 10^{-7}$ & $9.0 \cdot 10^{-6}$ & $4.9 \cdot 10^{-10}$ & $7.2 \cdot 10^{-9}$ & $6.6 \cdot 10^{-9}$ \\
$24 / 5$ & $1.0 \cdot 10^{-7}$ & $4.3 \cdot 10^{-6}$ & $7.2 \cdot 10^{-10}$ & $6.6 \cdot 10^{-9}$ & $8.3 \cdot 10^{-9}$ \\
$28 / 5$ & $5.4 \cdot 10^{-8}$ & $2.4 \cdot 10^{-6}$ & $6.8 \cdot 10^{-10}$ & $9.9 \cdot 10^{-9}$ & $8.5 \cdot 10^{-9}$ \\
$32 / 5$ & $1.6 \cdot 10^{-7}$ & $6.6 \cdot 10^{-6}$ & $6.3 \cdot 10^{-10}$ & $1.0 \cdot 10^{-8}$ & $8.5 \cdot 10^{-9}$ \\
$36 / 5$ & $2.3 \cdot 10^{-7}$ & $8.7 \cdot 10^{-6}$ & $6.0 \cdot 10^{-10}$ & $1.0 \cdot 10^{-8}$ & $8.5 \cdot 10^{-9}$ \\
8 & $2.9 \cdot 10^{-7}$ & $9.6 \cdot 10^{-6}$ & $5.8 \cdot 10^{-10}$ & $1.0 \cdot 10^{-8}$ & $8.4 \cdot 10^{-9}$ \\
\hline
\end{tabular}

there no exists an analytic terms in the errors, that is, we obtain the best analytic approximate solution.
TABLE 9: Values of $F^{\prime \prime}(0)$ and $F(\infty)$ for $\beta=0.5, M=0.75$, and $R=0.25$.

\begin{tabular}{lccc}
\hline Equation & $(46)$ & $(49)$ & $(55)$ \\
\hline$F_{\text {numeric }}^{\prime \prime}(0)$ & -1.6610234543 & -1.6610234543 & -1.6610234543 \\
$\bar{F}_{\text {OHAM }}^{\prime \prime}(0)$ & -1.6610517418 & -1.6608631140 & -1.6608887452 \\
$F_{\text {numeric }}(\infty)$ & 0.8305852596 & 0.8305852596 & 0.8305852596 \\
$\bar{F}_{\text {OHAM }}(\infty)$ & 0.8305852518 & 0.8305846668 & 0.8305850069 \\
\hline
\end{tabular}

TABLE 10: Values of $F^{\prime \prime}(0)$ and $F(\infty)$ for $\beta=0.5, M=0.75$, and $R=-2$.

\begin{tabular}{lccc}
\hline Equation & $(60)$ & $(61)$ & $(62)$ \\
\hline$F_{\text {numeric }}^{\prime \prime}(0)$ & -3.4445300498 & -3.4445300498 & -3.4445300498 \\
$\bar{F}_{\text {OHAM }}^{\prime \prime}(0)$ & -3.4445331335 & -3.4445928699 & -3.4446421233 \\
$F_{\text {numeric }}(\infty)$ & -1.7062261065 & -1.7062261065 & -1.7062261065 \\
$\bar{F}_{\text {OHAM }}(\infty)$ & -1.7062261070 & -1.7062260957 & -1.7062260959 \\
\hline
\end{tabular}

For all 12 approximate solutions (46)-(57) and for $n=30$ points, all errors pass both tests (e.g., we obtain \{1.87448, $1.87448,5.86776,2.13935,1.48597,1.68839\}$ for Barlett's test and respectively the values $\{1.81622,1.81622,1.92555,2.64548$, $1.83306,1.96373\}$ for Durbin-Wattson test).

\section{Conclusions}

The optimal homotopy asymptotic method is employed to propose new analytic approximate solutions for the upperconvected Maxwell fluid over a porous stretching plate. Our procedure is valid even if the nonlinear differential equation does not contain any small or large parameters. In construction of the homotopy appear some distinctive concepts such as the auxiliary functions $H_{1}\left(\eta, C_{i}\right), H_{2}\left(\eta, C_{i}\right), \ldots$, the linear operator $L$, and several optimal convergence-control parameters $C_{1}, C_{2}, \ldots$ which ensure a fast convergence of the all solutions. The examples presented in this work lead to the conclusion that the obtained results are of the exceptional accuracy using only one iteration. The OHAM provides us with a simple and rigorous way to control and adjust the convergence of the solutions through the auxiliary functions $H_{1}\left(\eta, C_{i}\right), H_{2}\left(\eta, C_{i}\right), \ldots$, involving several parameters $C_{1}, C_{2}, \ldots$ which are optimally determined. Actually, the capital strength of OHAM is its fast convergence, which proves that our procedure is very efficient in practice.

\section{Conflict of Interests}

The authors declare that there is no conflict of interests regarding the publication of this paper.

\section{References}

[1] B. C. Sakiadis, "Boundary layer behaviour an continuous solid surface I: boundary layer equations for two dimensional and axisymmetric flow," AIChEJ, vol. 7, no. 1, pp. 26-28, 1961.

[2] B. C. Sakiadis, "Boundary layer behaviour an continuous solid surface II: boundary layer on a continuous flat surface," AIChEJ, pp. 221-225, 1961. 
[3] N. Phan-Thien, "Plane and axi-symmetric stagnation flow of a Maxwellian fluid," Rheologica Acta, vol. 22, no. 2, pp. 127-130, 1983.

[4] R. Zheng, N. Phan-Thien, and R. I. Tanner, "On the flow past a sphere in a cylindrical tube: limiting Weissenberg number," Journal of Non-Newtonian Fluid Mechanics, vol. 36, pp. 27-49, 1990.

[5] K. Sadeghy, A.-H. Najafi, and M. Saffaripour, "Sakiadis flow of an upper-convected Maxwell fluid," International Journal of Non-Linear Mechanics, vol. 40, no. 9, pp. 1220-1228, 2005.

[6] T. Hayat, Z. Abbas, and M. Sajid, "Series solution for the upperconvected Maxwell fluid over a porous stretching plate," Physics Letters A: General, Atomic and Solid State Physics, vol. 358, no. 5-6, pp. 396-403, 2006.

[7] T. Hayat and M. Sajid, "Homotopy analysis of MHD boundary layer flow of an upper-convected Maxwell fluid," International Journal of Engineering Science, vol. 45, no. 2-8, pp. 393-401, 2007.

[8] Z. Abbas, T. Hayat, M. Sajid, and S. Asghar, "Unsteady flow of a second grade fluid film over an unsteady stretching sheet," Mathematical and Computer Modelling, vol. 48, no. 3-4, pp. 518526, 2008.

[9] Z. Abbas, Y. Wang, T. Hayat, and M. Oberlack, "Mixed convection in the stagnation-point flow of a Maxwell fluid towards a vertical stretching surface," Nonlinear Analysis: Real World Applications, vol. 11, no. 4, pp. 3218-3228, 2010.

[10] T. Hayat, M. Awais, M. Qasim, and A. A. Hendi, "Effects of mass transfer on the stagnation point flow of an upper-convected Maxwell (UCM) fluid," International Journal of Heat and Mass Transfer, vol. 54, no. 15-16, pp. 3777-3782, 2011.

[11] A. Ishak, R. Nazar, N. M. Arifin, and I. Pop, "Dual solutions in mixed convection flow near a stagnation point on a vertical porous plate," International Journal of Thermal Sciences, vol. 47, no. 4, pp. 417-422, 2008.

[12] B. Sahoo, "Effects of slip, viscous dissipation and Joule heating on the MHD flow and heat transfer of a second grade fluid past a radially stretching sheet," Applied Mathematics and Mechanics, vol. 31, no. 2, pp. 159-173, 2010.

[13] H. Schlichting, Boundary Layer Theory, McGraw-Hill, New York, NY, USA, 6th edition, 1964.

[14] V. Marinca and N. Herişanu, Nonlinear Dynamical Systems in Engineering, Springer, Heidelberg, Germany, 2011.

[15] V. Marinca, N. Herişanu, and I. Nemeş, "Optimal homotopy asymptotic method with application to thin film flow, Central European Journal of Physics, vol. 6, pp. 648-653, 2008.

[16] V. Marinca, N. Herişanu, C. Bota, and B. Marinca, "An optimal homotopy asymptotic method applied to the steady flow of a fourth-grade fluid past a porous plate," Applied Mathematics Letters, vol. 22, no. 2, pp. 245-251, 2009.

[17] V. Marinca and N. Herişanu, "An optimal homotopy asymptotic approach applied to nonlinear MHD Jeffery-Hamel flow," Mathematical Problems in Engineering, vol. 2011, Article ID 169056, 16 pages, 2011.

[18] V. Marinca and N. Herişanu, "An optimal homotopy perturbation approach to thin film flow of a fourth grade fluid," in Proceedings of the International Conference of Numerical Analysis and Applied Mathematics (ICNAAM '12), pp. 23832386, September 2012.

[19] L. Elsgolts, Differential Equations and the Calculus of Variations, Mir Publishers, Moscow, Russia, 1973. 


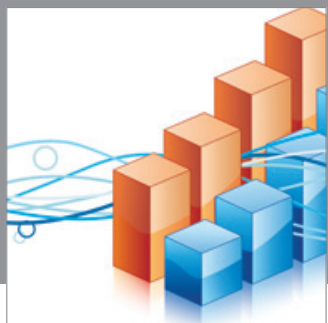

Advances in

Operations Research

mansans

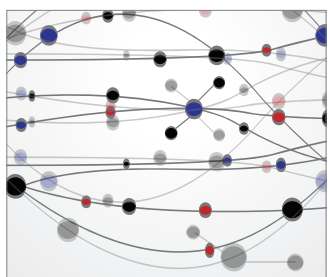

The Scientific World Journal
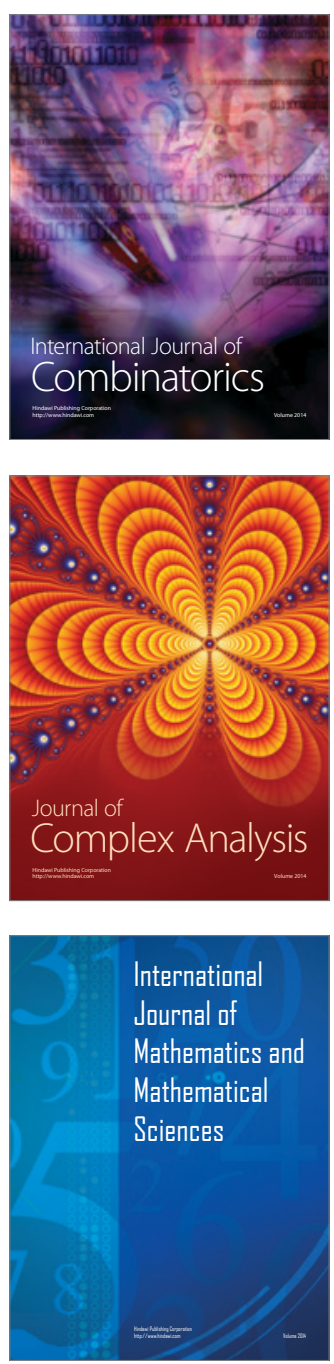
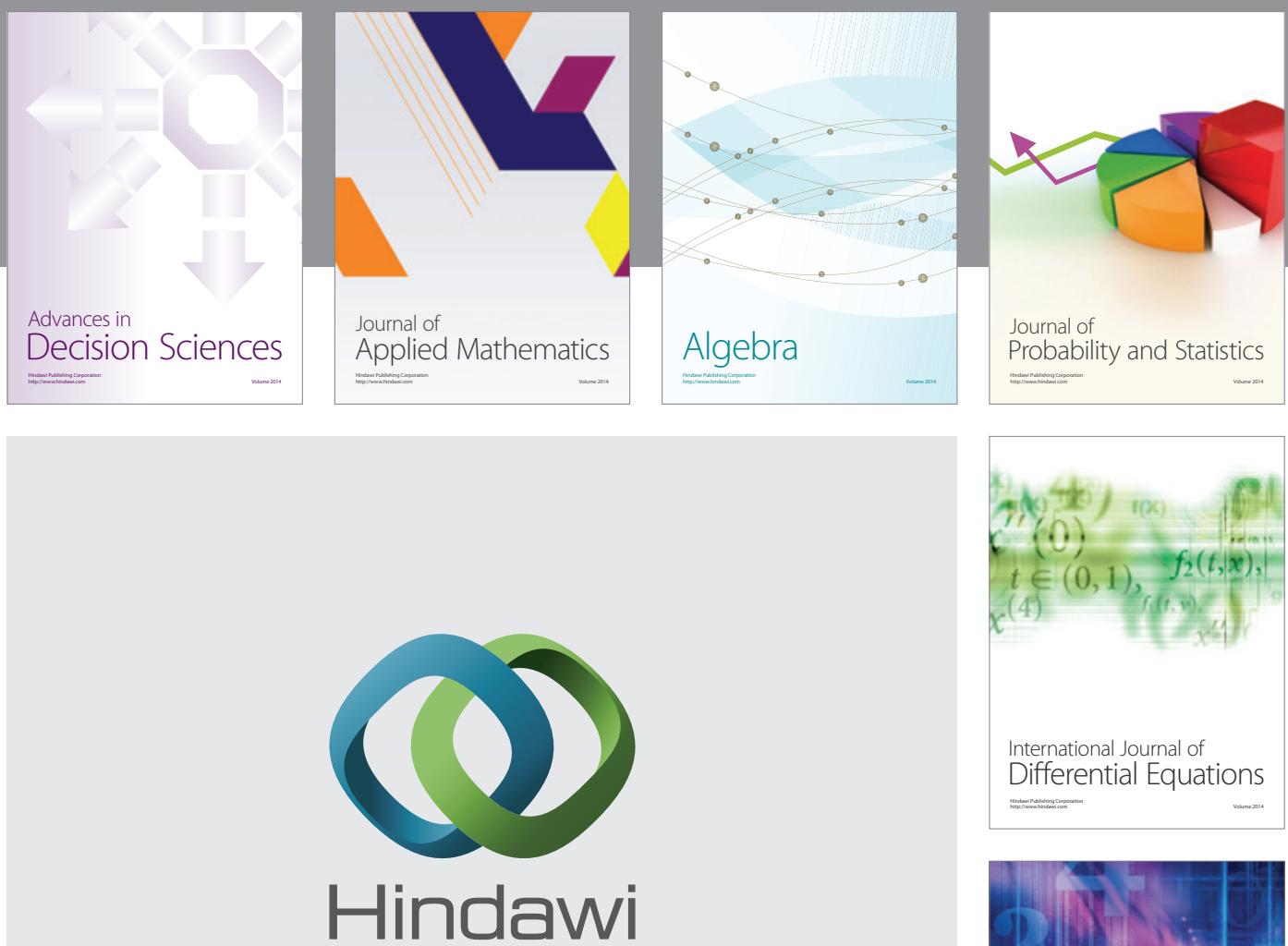

Submit your manuscripts at http://www.hindawi.com
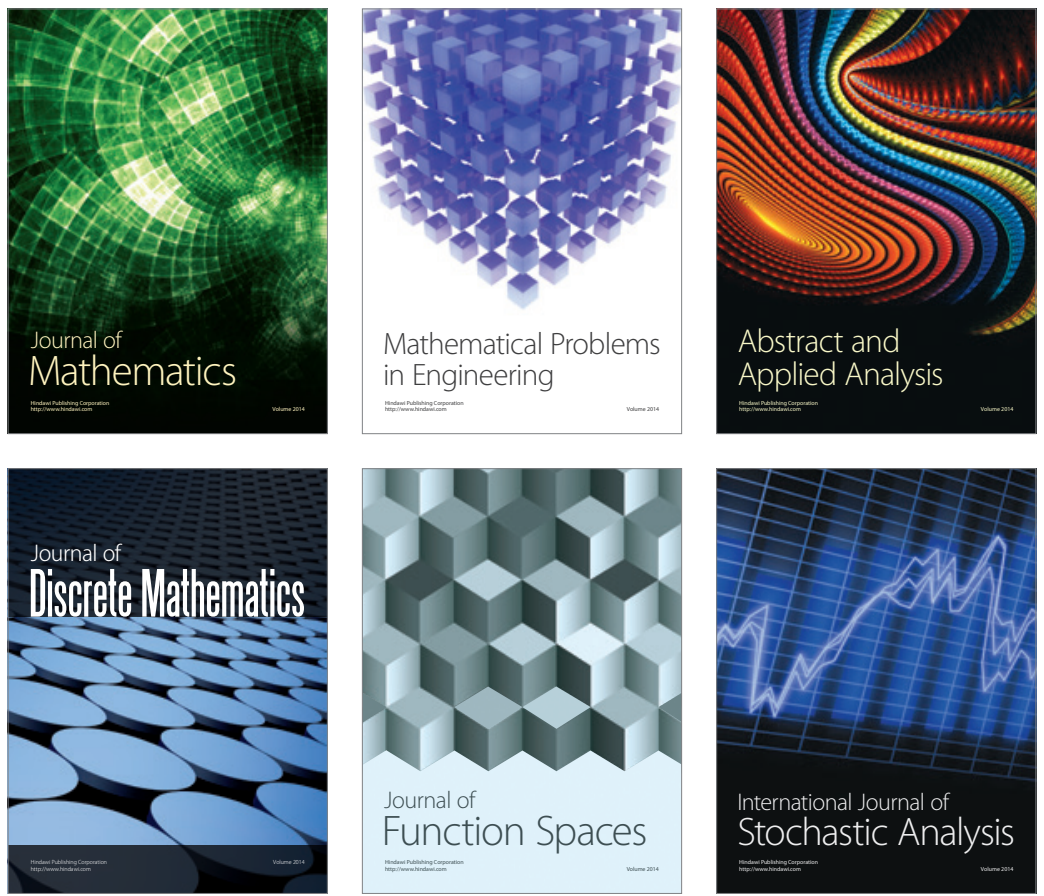

Journal of

Function Spaces

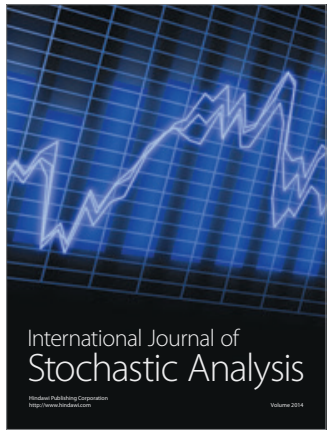

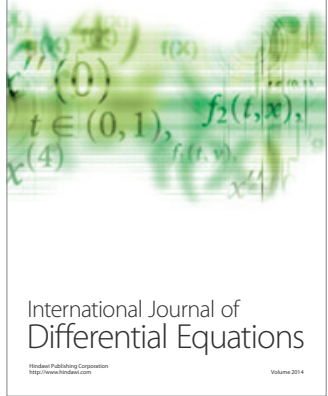
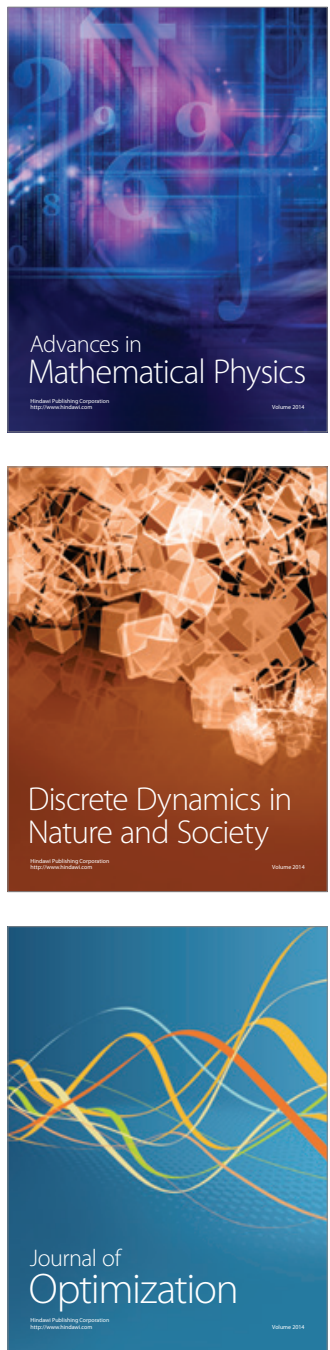